\title{
Chemical Structure and Intermolecular Cooperativity: Dielectric Relaxation Results
}

\author{
K. L. Ngai and C. M. Roland* \\ Naval Research Laboratory, Washington, D.C. 20375-5320 \\ Received May 3, 1993; Revised Manuscript Received September 15, $1993^{\circ}$
}

\begin{abstract}
The effect of chemical structure on the segmental relaxation behavior is examined for a wide range of polymers. Both the time and temperature dependence of the glass transition dispersion in the dielectric loss spectrum are shown to be correlated with the degree to which local structure engenders steric constraints on the relaxation from neighboring nonbonded segments. The polymers studied exhibit a range of segmental relaxation behavior. For polymers with smooth, compact, symmetrical chain backbones, segmental relaxation times have a near Arrhenius temperature dependence, representing the limiting-type behavior associated with relatively unconstrained ("Debye") relaxation. Polymers having less flexible backbones and/ or sterically-hindering pendant groups exhibit broad segmental dispersions and temperature dependencies near the extreme fragile edge; these characteristics reflect segmental relaxation that is strongly intermolecularly cooperative. The data for polymers parallel observations made on small-molecule glass-forming liquids, whose chemical structure similarly governs the strength of the intermolecular coupling, and thus the time and temperature dependence of segmental relaxation.
\end{abstract}

\section{Introduction}

Segmental relaxation, occurring near the glass transition temperature, involves localized motion of several backbone bonds; thus, the shape of the viscoelastic dispersion is expected to reflect in some manner the local molecular structure. The glass transition temperature itself has been related to chemical structure. ${ }^{1-4}$ In addition, for both small-molecule liquids ${ }^{5-10}$ and polymers, ${ }^{11-16}$ correlations have been demonstrated between the temperature and time dependencies of segmental relaxation. However, attempts to relate these characteristics of the relaxation (i.e., the degree of nonexponentiality, or equivalently the shape of the segmental relaxation function, and the temperature dependence of the relaxation time) to chemical structure are scarce.

Tobolsky, Aklonis, and co-workers have developed a model ${ }^{17,18}$ to describe the relaxation behavior of various polymers in the glass transition zone in terms of the severity of intra- and interchain interactions. An empirical correlation has been proposed for the time dependence of segmental relaxation and a polymer's cohesive energy density, flexibility, and available free volume. ${ }^{19}$ Some experimental studies have found no connection between the chemical structure of certain polymers and the shape of the segmental relaxation function, ${ }^{20}$ while, for example, hydrogenation of polystyrene was reported to decrease the segmental relaxation rate. ${ }^{21}$

The mechanism for segmental relaxation involves skeletal bond rotations, with motion over large length scales avoided by cooperative rotations of neighboring units along the chain. This intramolecular cooperativity gives rise to a segmental relaxation function having the Hall-Helfand ${ }^{22,23}$ or similar ${ }^{24,25}$ form. Polymers in dense phase have their motion further restricted by intermolecular cooperativity. ${ }^{26-32}$ At times sufficient for intermolecular interactions to manifest themselves, the motion of a segment becomes coupled to those of neighboring, nonbonded species. This retards the relaxation, to an extent dependent on the strength of the intermolecular constraints. In this paper we are primarily concerned with

Abstract published in Advance ACS Abstracts, November 1, 1993 intermolecular cooperativity arising from steric interferences, although, in general, polar forces, specific interactions, etc., can contribute. According to the coupling model of relaxation, ${ }^{26-29}$ for neat polymers at the times appropriate for most experimental measurements, the slowing down of segmental relaxation gives rise to a correlation function having the Kohlrausch-WilliamsWatts form 33,34

$$
E(t)=E(0) \exp \left[-\left(t / \tau^{*}\right)^{1-n}\right]
$$

The temperature-dependent relaxation time $\tau^{*}$ is given by $^{26-29}$

$$
\tau^{*}=\left([1-n] \omega_{\mathrm{c}}^{n} \tau_{0}\right)^{1 /(1-n)}
$$

where $\tau_{0}$ is one of the Hall-Helfand (intermolecularly uncorrelated) relaxation times. ${ }^{15,16}$ Recent quasi-elastic neutron scattering experiments, ${ }^{35}$ as well as molecular dynamics simulations, ${ }^{36}$ have confirmed the existence of a temperature-independent crossover time, $\omega_{\mathrm{c}}{ }^{-1}$, in eq 2 , at which segmental relaxation assumes the KWW form. The parameter $n$, characterizing the degree of nonexponentiality of the relaxation function or the spectral width of the corresponding dispersion, is a measure of the strength of the intermolecular constraints on segmental relaxation. All derivations of the coupling model have either explicitly or implicitly shown that the nonexponentiality parameter $n$ in eqs 1 and 2 is proportional to the strength of the constraints or interactions with nonbonded neighboring segments. This interpretation is unique to the coupling model and, of course, is why $n$ is referred to as the coupling parameter. The value of this parameter $(0 \leq n<1)$ for a given species must depend on molecular structure because the latter ultimately determines the intermolecular interactions. However, the complexity of cooperative dynamics in dense liquids and polymers precludes direct calculation; $n$ must be deduced from experiment.

From eq 2 it is seen that any temperature dependence of $\tau_{0}$, pertaining to conformational transition rates of an isolated chain, will be amplified in the dense phase by the $1 /(1-n)$ power. This means that the temperature dependence of segmental relaxation observed for a neat polymer will parallel the magnitude of its coupling parameter. ${ }^{6,7,11}$ Thus, the experimentally observed cor- 
relation between time and temperature dependencies ${ }^{5-16}$ is predicted by the coupling model of relaxation.

Many polymers, especially those having simple chemical structures and hence weak intermolecular coupling, are semicrystalline. The restriction on the motion of amorphous chain segments near crystallites causes inhomogeneous broadening of the segmental relaxation dispersion. ${ }^{37-40}$ The dispersion no longer has the KWW form (eq 1), precluding determination of $n$ by direct fitting. In a recent work $\mathrm{k}^{41}$ we demonstrated that crystallinity has an insignificant effect on the measured temperature dependence of the segmental relaxation time, at least when the temperature is normalized by the glass transition temperature, $T_{g}$, of the polymer. Since the temperature dependence of the relaxation is still dominated by intermolecular cooperativity, its correlation with the intermolecular coupling strength of segmental relaxation (i.e., the magnitude of $n$ ) is retained. ${ }^{41}$ This provides a means of determining $n$, and hence assessing the degree of intermolecular cooperativity and its dependence on chemical structure, even for highly crystalline polymers. A plot of the relaxation time or transport coefficient versus $T_{\mathrm{g}}$-scaled temperature was first introduced by Laughlin and Uhlmann ${ }^{42}$ and exploited by Angell, ${ }^{43,44}$ who interpreted the sensitivity of the scaled temperature variation in terms of either the degradation of the structure with temperature ("fragility") or the nature of a multidimensional free energy hypersurface. For this reason, semilogarithmic depictions of $\tau$ versus $T_{\mathrm{g}} / T$ are customarily referred to as fragility plots. We have suggested ${ }^{12}$ a more appropriate name, at least for polymers, is the cooperativity plot. The reason for this suggestion is obvious from the discussion above.

In this paper we present segmental relaxation data on a variety of polymers, both amorphous and semicrystalline, intended to demonstrate a relationship between chemical structure and relaxation behavior. Specifically, it will be shown that there is less constraint on segmental relaxation from interactions with neighboring, nonbonded segments in polymers with smoother, less polar, more compact, symmetric or flexible-chain backbones and/or having less sterically-hindering pendant groups. This correlation of intermolecular cooperativity with chemical structure has been demonstrated previously for polybutadienes ${ }^{12}$ and epoxidized polyisoprenes. ${ }^{16}$ The strength of the intermolecular coupling is deduced from the normalized temperature dependence of the segmental relaxation time and also, for amorphous polymers, from the spectral width of the segmental relaxation dispersion. Using this approach, the present study provides an interpretation of how chemical structure governs the magnitude of $n$ (or the KWW stretch exponent, $1-n$ ).

\section{Results}

Polyethers. Cooperativity plots, in the form of $T_{\mathrm{g}}$ scaled Arrhenius plots of the frequency of the maximum in the dielectric loss from segmental motion, measured for poly(oxymethylene) (POM), poly(tetrahydrofuran) (PTHF), poly (acetaldehyde) (PAA), and poly(propylene oxide) (PPO) are displayed in Figure 1. POM and PTHF are semicrystalline, while PAA and PPO are amorphous. The structures of the monomer units for these four polyethers are also illustrated in Figure 1. Dielectric measurements are usually performed in the frequency range of $10<f<10^{6} \mathrm{~Hz}$, and it is customary to define $T_{\mathrm{g}}$ operationally as the temperature at which the segmental relaxation time equals $100 \mathrm{~s}$. However, to avoid possible errors caused by an extrapolation of the dielectric relaxation time to $100 \mathrm{~s}$, we define $T_{\mathrm{g}}$ instead to be the temperature at which $\tau^{*}$ reaches $1 \mathrm{~s}$. The segmental

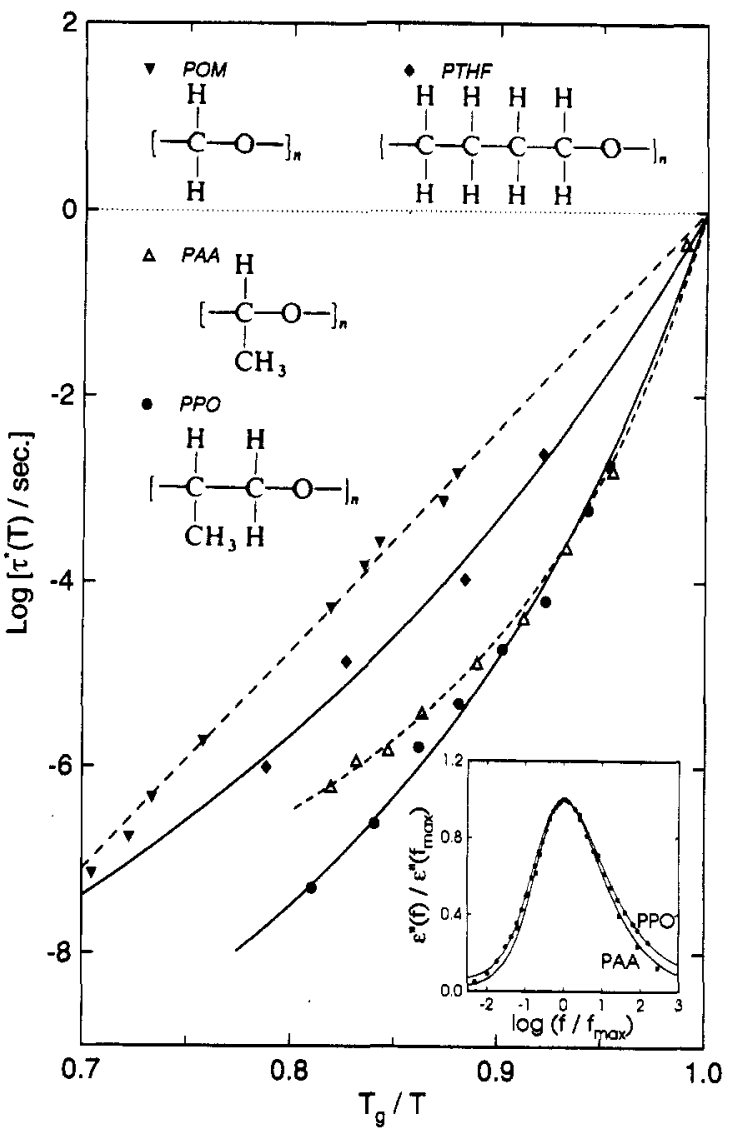

Figure 1. Segmental relaxation time as a function of $T_{8}$ normalized temperature for poly (oxymethylene) $\left(T_{\mathrm{g}}=183 \mathrm{~K}\right.$ ), poly(tetrahydrofuran) (or poly(tetramethylene oxide), $T_{8}=178$ $\mathrm{K})$, poly(acetaldehyde) $\left(T_{\mathrm{g}}=253 \mathrm{~K}\right)$, and poly(propylene oxide) $\left(T_{\mathrm{g}}=205 \mathrm{~K}\right)$. Intermolecular cooperativity increases with an increase in the relative number in the backbone of the methylene units, which are less flexible than the ether linkage. The inset shows the corresponding dispersions in the dielectric loss spectrum for the two amorphous polymers, PPO and PAA. Except where otherwise noted, in this and other figures, the segmental relaxation time, $\tau^{*}$, is defined to equal $\left(2 \pi f_{\text {mas }}\right)^{-1}$, where $f_{\max }$ is the frequency of the peak maximum, while $T_{\mathrm{B}}$ is the temperature at which $\tau^{*}=1 \mathrm{~s}$.

relaxation time of the PPO is seen in Figure 1 to exhibit the strongest dependence on temperature, implying strongest intermolecular coupling. The POM segmental relaxation is the least temperature sensitive, as reflected by a nearly Arrhenius temperature dependence. The temperature sensitivity of the segmental relaxation increases in the order POM $<$ PTHF $<$ PAA $<$ PPO. Let us discuss this trend on the basis of the dependence of intermolecular cooperativity on chemical structure.

PTHF contains the same main-chain $\mathrm{C}-\mathrm{O}$ bonds found in POM, but the ether linkages are interrupted by a sequence of three $\mathrm{C}-\mathrm{C}$ bonds. Rotation of the $\mathrm{C}-\mathrm{C}$ bonds is restricted to certain conformational transitions, unlike the free rotation afforded by the $\mathrm{C}-\mathrm{O}$ linkage. The additional intramolecular constraints of $\mathrm{C}-\mathrm{C}$ bonds in comparison to the $\mathrm{C}-\mathrm{O}$ bond will enhance the intermolecular coupling or cooperativity because some of the reorientations of the $\mathrm{C}-\mathrm{O}$ bonds are not possible for $\mathrm{C}-\mathrm{C}$ bonds. Thus, intermolecular cooperativity should be enhanced in PTHF when compared with POM. This is indeed the case, as demonstrated by the greater temperature sensitivity of PTHF's segmental relaxation (Figure 1), implying greater intermolecular coupling. Both POM and PTHF being semicrystalline, the dielectric dispersion itself cannot be usefully analyzed to obtain the coupling parameter directly, since the peak is broadened not only by intermolecular cooperativity but also by the presence 
of crystallinity. However, quasi-elastic neutron scattering data $^{45,46}$ and its interpretation by the coupling model ${ }^{47}$ make possible a determination of the coupling parameter even for semicrystalline polymers. According to ref 39 , the momentum transfer, $Q$, dependence of the local segmental relaxation time is a power law given by $Q^{2 /(1-n)}$. For PTHF, the experimentally observed $Q$-dependence is $Q^{3.1}$ at $346 \mathrm{~K}$ and $Q^{3.5}$ at $423 \mathrm{~K}$, ${ }^{45}$ whereby we deduce the coupling parameter for PTHF to be

$$
n_{\text {PTHF }}=0.37 \text { and } 0.43
$$

respectively. From the discussion immediately above and given the lesser temperature sensitivity of POM, we expect the coupling parameter of POM to be even smaller, i.e.

$$
n_{\text {POM }}<n_{\text {PTHF }}
$$

Compared with the known values of the coupling parameter of other amorphous polymers, usually having more complicated monomer structures, the small values for PTHF indicate a modest degree of intermolecular coupling of the segmental relaxation, consistent with the very flexible either linkage in the P'THF backbone. It is possible that P'THF is less polar than POM, and conceivably this weakens the intermolecular coupling. However, the stronger temperature sensitivity of PTHF versus POM (Figure 1) reveals that this contribution is unimportant.

Closely related in structure to POM is amorphous PAA (Figure 1) which has a pendant methyl group on every skeletal carbon. Steric interactions between nonbonded neighbors involving this pendant group are expected to more intermolecularly constrain the segmental relaxation. Since PAA is amorphous, the dielectric loss and dispersion shown in the inset of Figure 1 can be used to estimate $n$. From the fit of the dielectric loss as a function of frequency to that expected from the KWW correlation function given by eq 1 (Figure 1), we find

$$
n_{\mathrm{PAA}}=0.47
$$

This result together with eq 3 and inequality eq 4 confirms the result

$$
n_{\mathrm{POM}}<n_{\mathrm{PAA}}
$$

deduced from the greater temperature sensitivity of PAA's segmental relaxation (Figure 1).

From the discussion above concerning PTHF and POM, we anticipate that the additional carbon in the backbone of PAA to yield PPO will increase the cooperativity, although the enhancement may not be large. The segmental relaxation of $\mathrm{PPO}$, measured using dielectric spectroscopy, ${ }^{48,49}$ is found to be only slightly more dependent on normalized temperature (i.e., more cooperative) than that of PAA (Figure 1). This is in accord with the breadth of their dielectric loss spectra (Figure 1, inset). The coupling parameter determined from fitting the dielectric dispersion of PPO with the KWW correlation function has the value

$$
n_{\mathrm{PPO}}=0.51
$$

(consistent with a very recent result $n_{\mathrm{PPG}}=0.50$ for poly(propylene glycol) (PPG)). This is indeed slightly larger than $n_{\text {PAA. }}$. At least for these polyoxides, for which $n$ can be directly determined from experimental data (i.e., the shape of the viscoelastic dispersion for the amorphous polymers or the $Q$-dependence of the relaxation time from quasi-elastic neutron scattering), the correlations among monomer structure, frequency dependence, and $T_{\mathrm{g}}$-scaled temperature dependencies are all directly corroborated.

This correlation of structure with temperature dependence implies that the cooperativity curve of poly(ethylene

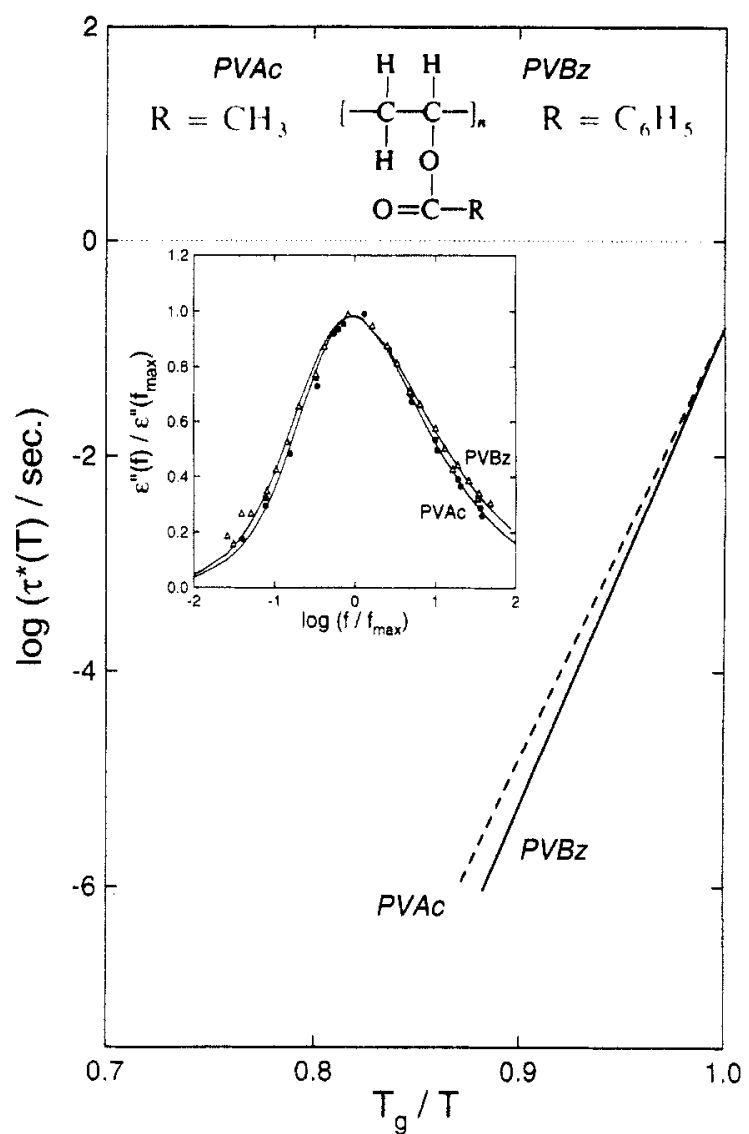

Figure 2. Cooperativity plots for poly(vinyl acetate) and poly(vinyl benzoate) along with the corresponding segmental relazation dispersions. Due to the limited data available, $T_{\mathrm{g}}(=318$ $\mathrm{K}$ and $359 \mathrm{~K}$ for PVAc and PVBz, respectively) in this figure only is taken to be the temperature at which $f_{\max }=1 \mathrm{~Hz}$. The bulky phenyl group in the PVBz is associated with a broadening of the peak and a steeper temperature dependence. Both these effects are ascribed to stronger intermolecular coupling.

oxide) (PEO) will fall between that of POM and PTHF in Figure 1. Unfortunately, at lower temperatures the dielectric dispersion of the highly crystalline PEO is extremely broad, ${ }^{37,50}$ introducing a large error in the determination of the peak maximum. Also, the use of mechanical measurements to extrapolate the dielectric data to lower frequencies ${ }^{37,50}$ can be misleading. For example, in PTHF the frequency of the maximum in the dielectric loss can at equal temperatures differ by 4 orders of magnitude from the corresponding peak in the mechanical loss modulus. ${ }^{38}$ An analysis of this strong probe dependence of the relaxation behavior, previously reported for PTHF and POM, ${ }^{38}$ can in fact reveal interesting physics which will be the subject of a future publication. ${ }^{51}$ For the present purpose, we note only that reliance on the limited higher temperature dielectric relaxation data of $\mathrm{PEO}^{50}$ would give a result consistent with the correlation suggested by the four polymers of Figure 1.

Poly(vinyl esters). Poly(vinyl acetate) (PVAc) and poly (vinyl benzoate) (PVBz) are two members of the poly(vinyl esters) family; the structures of their chain units are closely related as illustrated in Figure 2. The bulkier side group of $\mathrm{PVBz}$ is expected to effect stronger intermolecular cooperativity than in PVAc. Although the increase in cooperativity may not be large because the structural variation occurs only in the pendant group, the directional changes in going from PVAc to $\mathrm{PVBz}$ can be unambiguously predicted, to wit, an increase in the breadth of the dispersion (i.e., in the coupling parameter) and a greater temperature sensitivity in the $T_{\mathrm{g}}$-scaled temperature dependence plot. From a comparison of dielectric 


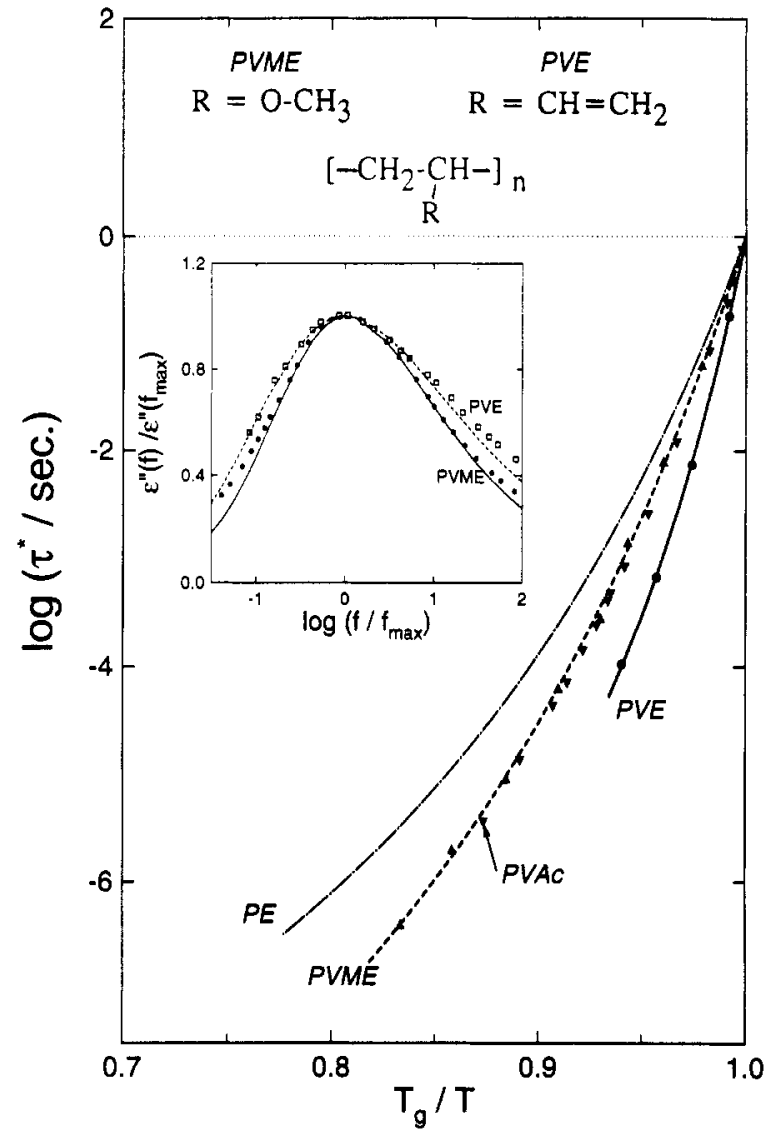

Figure 3. $T_{\mathrm{g}}$-scaled temperature dependence and the segmental dispersions for poly(vinyl methyl ether) $\left(T_{\mathrm{g}}=256 \mathrm{~K}\right)$ and poly(vinylethylene) $\left(T_{\mathrm{g}}=271 \mathrm{~K}\right)$. The less flexible pendant group in the latter effects stronger intermolecular coupling and hence a broader dispersion and steeper cooperativity plot. Cooperativity data for poly(vinyl acetate) and polyethylene are shown for comparison.

data of PVAc and PVBz, ${ }^{52}$ we can see that indeed these two characteristics of segmental relaxation change in the expected way (Figure 2). KWW fits of the dielectric loss spectra of the two polymers give $n_{\mathrm{PVAc}}=0.41$ and $n_{\mathrm{PVBz}}$ $=0.46$, verifying that

$$
n_{\mathrm{PVAc}}<n_{\mathrm{PVBz}}
$$

The frequency of the maximum dielectric loss, $f_{\max }$, in ref 44 follows an Arrhenius law over the limited frequency range used for both PVAc and PVBz. Actually, it is known that, over a more extended frequency window, the temperature dependence of PVAc ${ }^{53,54}$ has the Vogel-Fulcher form. We do not want to extrapolate the measured relaxation times to $1 \mathrm{~s}$ in order to determine $T_{\mathrm{g}}$, because large error could be introduced. For this reason, in comparing the $T_{\mathrm{g}}$-scaled temperature dependence of the relaxation time of PVAc and PVBz using the dielectric data of ref 44, we define $T_{\mathrm{g}}$ to be the temperature at which $\log \left(f_{\max } / \mathrm{Hz}\right)=1$ or $\tau^{*}=1 /(2 \pi \times 10) \mathrm{s}$. Figure 2 is the only exception in this paper from the convention that $\tau^{*}\left(T_{\mathrm{g}}\right)$ $=1 \mathrm{~s}$.

Polymers with a Polyethylene Backbone. Our previous work on semicrystalline polymers ${ }^{41}$ has shown that the $T_{\mathrm{g}}$-scaled temperature dependence of the segmental relaxation time determined from the peak of the dielectric loss can be used as a measure of the degree of cooperativity, even though the dispersion itself is inhomogeneously broadened. Thus, we can interpret the temperature dependence of the dielectric relaxation time for linear polyethylene (PE), shown in Figure 3, in terms of the strength of the intermolecular coupling, even though the polymer is highly crystalline. The reason for considering polyethylene here is to contrast its structure with

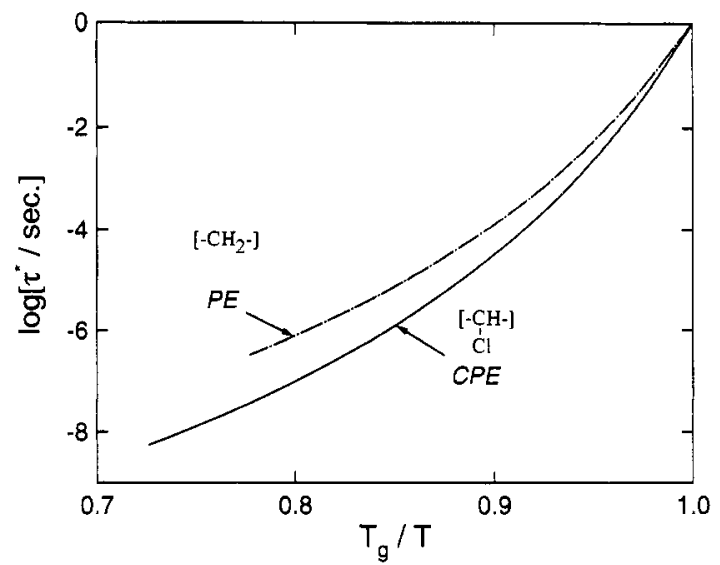

Figure 4. Cooperativity plots for polyethylene $\left(T_{\mathrm{g}}=200 \mathrm{~K}\right)$ and chlorinated polyethylene (a random copolymer, $T_{\mathrm{g}}=312 \mathrm{~K}$, containing the repeat units shown above). The introduction of the large, polar chlorine atom enhances intermolecular coupling, as reflected in a stronger dependence of $\tau^{*}$ on $T_{\mathrm{g}}$-scaled temperature.

that of poly(vinylethylene) (PVE). PVE (or 1,2-polybutadiene) has a simple backbone like polyethylene, but on every other skeletal carbon there is a pendant vinyl moiety. These relatively inflexible vinyl carbons project over $3 \AA$ from the chain backbone and thus sweep out a large volume during the course of conformational transitions (Figure 3). For this reason, we expect stronger intermolecular coupling (higher degree of cooperativity) in PVE in comparison to $\mathrm{PE}$. Indeed, as seen by inspection of Figure 3, the $T_{\mathrm{g}}$-scaled temperature dependence of the relaxation time of polyethylene is much weaker than that of PVE.

In Figure 4 the $T_{\mathrm{g}}$-scaled temperature dependence of $\mathrm{PE}$ is compared to a polyethylene (CPE) in which $37 \%$ of the chain units have been chlorinated. ${ }^{55}$ Steric effects arising from the pendant chlorine atoms, as well as the enhanced intermolecular interactions associated with their polarity, should increase the degree of intermolecular coupling relative to $\mathrm{PE}$. This expectation is borne out by the steeper cooperativity curve in Figure 4 for CPE than for PE. The dispersion of the CPE is also very broad, congruent with strong intermolecular coupling. We note in passing, however, that in random copolymers the possibility exists for a special form of inhomogeneous broadening. Since the backbone structure is inhomogeneous, there may be different $\tau_{0}$, corresponding to different conformational transition rates for the different chain units. This contribution to the breadth of the dispersion is the subject of current studies but beyond the scope of the present work.

The strong intermolecular coupling described above for PVE is attributed to steric encounters of the vinyl groups, mutually and with the chain backbone. This can be contrasted with 1,4-polybutadiene (PBD), which has only protons attached to the carbon backbone and thus presumably a much weaker coupling. In random copolymers of polybutadiene, an increase in the concentration of chain units having the 1,2-addition product should enhance the couplings between the locally rearranging chain segments, increasing the coupling parameter. This leads to predictions regarding the segmental relaxation properties. In particular, the breadth of the dispersion and the sensitivity of the $T_{\mathrm{g}}$-scaled temperature dependence of the relaxation time will both increase with vinyl (the 1,2-addition product) content. These predictions have been verified by mechanical measurements on a series of polybutadienes with different 1,2 -content. ${ }^{12}$ Since this paper considers only dielectric relaxation from segmental motion, we have made dielectric measurements in one PBD 


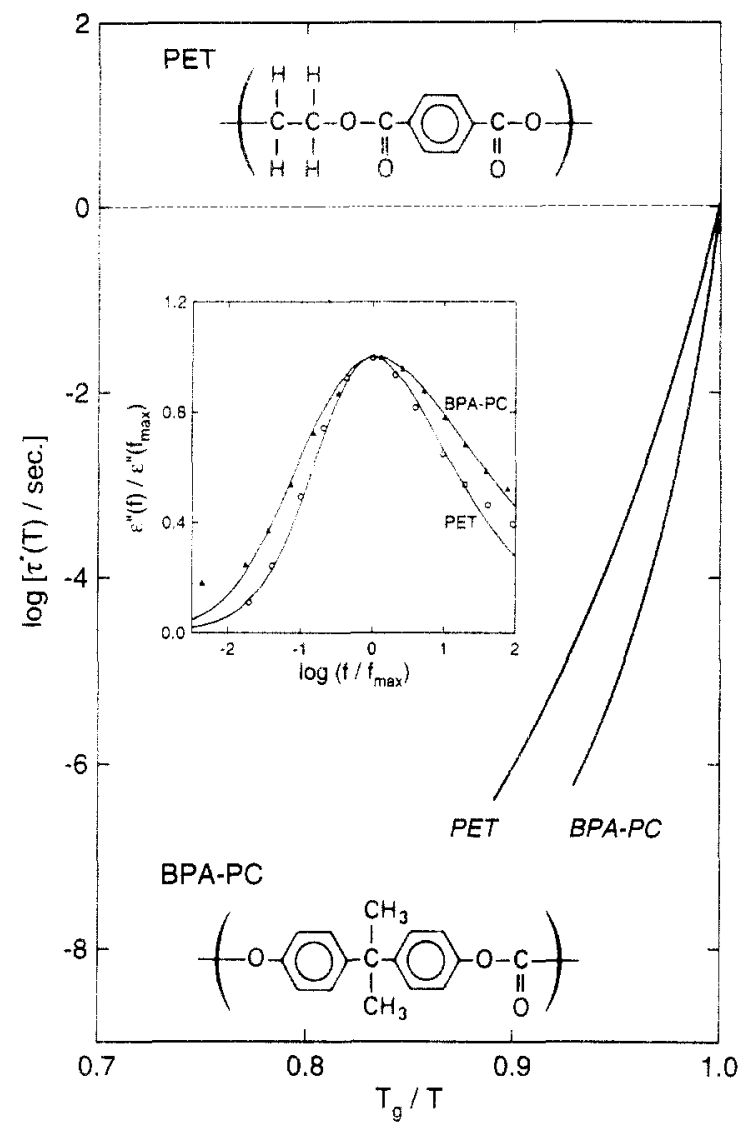

Figure 5. Cooperativity plots and segmental dispersions for polycarbonate synthesized from Bisphenol $\mathrm{A}\left(T_{\mathrm{g}}=419 \mathrm{~K}\right)$ and for poly (ethylene terephthalate) $\left(T_{\mathrm{g}}=353 \mathrm{~K}\right)$. The BPA-PC has a bulkier, less flexible backbone structure. The consequently more intermolecularly cooperative segmental relaxation results in a broader dispersion and stronger $T_{\mathrm{g}}$-scaled temperature dependence.

sample with a 1,2 content of $96 \%$. This particular sample was not studied in ref 11 by mechanical relaxation measurements. The dielectric dispersion noticeably broadens with decreasing temperature ${ }^{56}$ like that found by Mashimo and co-workers in PVAc. ${ }^{53,54}$ The dielectric loss peak measured at $0{ }^{\circ} \mathrm{C}$ can be well-fitted to eq 1 using $n_{\text {PVE }}=0.59$ (Figure 3). Also in Figure 3 is the corresponding dielectric data for poly(vinyl methyl ether) (PVME). ${ }^{57}$ The pendant moiety in PVME (Figure 3) is more flexible compared with that of PVE, and from this we expect intermolecular coupling to be reduced. This expected chemical structure dependence of the breadth of the dielectric dispersion is confirmed by the fit to eq 1 , which yields $n_{\text {PVME }}=0.52$ at $6^{\circ} \mathrm{C}$. Figure 3 also shows that the steepnesses of the $T_{\mathrm{g}}$-scaled temperature dependencies of PVE and PVME are consistent with the relative magnitude of their coupling parameters.

The comparison can be extended to PVAc, another vinyl polymer with the polyethylene backbone (structure shown in Figure 2). The pendant group in PVAc is even "bulkier" than that of PVE; however, the vinyl moiety of PVE is more sterically constraining, and this is evidently the determining factor for the degree of intermolecular coupling. We again conclude that intermolecular coupling is stronger in PVE than in PVAc, as can be verified by comparing the results for PVE with data obtained for PVAc, ${ }^{53,54}$ the latter taken over a wide range extending to very low frequencies (see Figure 3).

Polycarbonate versus Poly(ethylene terephthalate). Polycarbonates synthesized from Bisphenol A (BPA-PC) have bulkier and less flexible chain units (Figure 5) in comparison to all the polymers discussed so far. On the basis of this difference in molecular structure, we may conclude that its coupling parameter, $n_{\mathrm{PC}}$, is larger than any of the coupling parameters of the polymers considered above and also that the $T_{\mathrm{g}}$-scaled temperature dependence of its relaxation time is considerably stronger. These predictions are verified by the dielectric data of BPA polycarbonate ${ }^{58,59}$ and tetramethyl polycarbonate (TMPC). ${ }^{60}$ A more interesting comparison with BPA-PC is poly (ethylene terephthalate) (PET), whose respective chain units bear some resemblance to each other (Figure 5). The essential difference is the additional phenyl ring in BPA-PC replacing the more flexible and compact ethylene unit in PET. Naturally, we then expect stronger intermolecular coupling in BPA-PC and the closely related TMPC than in PET. BPA-PC is amorphous and PET can be quenched into the amorphous state. For amorphous polymers, the breadth of the dielectric dispersion can be used as a measure of intermolecular coupling. The dielectric loss data of BPA-PC ${ }^{58,59}$ and amorphous PET ${ }^{40}$ are compared in the inset of Figure 5. Clearly the width of the dielectric loss peak of BPA-PC is larger than that of PET. From the fits to eq 1 (Figure 5), the coupling parameters are determined to have the values of $n_{\mathrm{PC}}=$ 0.64 and $n_{\mathrm{PET}}=0.52$; thus, $n_{\mathrm{PC}}>n_{\mathrm{PET}}$. The $T_{\mathrm{g}}$-scaled temperature dependencies of the relaxation time for BPAPC and PET are compared in Figure 5, which reveals the stronger variation for BPA-PC than for PET.

Universality of Relaxation Phenomenology. The examples above, contrasting polymers of similar chemical structure, are consistent with a correlation between chain structure (flexibility, steric hindrance, compactness, smoothness, and symmetry of the backbone and similar considerations for any pendant groups) and the degree of intermolecular cooperativity of the segmental relaxation. In particular, the focus has been on the relative effects of steric constraints on segmental relaxation. This assumes that the intermolecular potentials are similar in nature, since the magnitude of the intermolecular forces (arising from London dispersion forces, polar interactions, hydrogen bonding, complex formation, etc.) obviously will influence the segmental dynamics as well.

When the polymers under consideration are amorphous, the breadth of the segmental relaxation dispersion can be used directly to determine the degree of intermolecular coupling. The inhomogeneous broadening of this dispersion in semicrystalline polymers precludes a direct determination of $n$; nevertheless, the strength of the coupling can be judged from the temperature dependence of the relaxation time..$^{41}$ This is enormously useful, since those polymers expected to exhibit very weak intermolecular cooperativity, by virtue of simple, compact chain structures, are invariably crystalline.

In each of the examples given above we consider a class of polymers with chemical structures that bear a logical relation to each other, and trends in the variation of intermolecular cooperativity can be predicted unequivocally. The changes in the degree of cooperativity are manifested by corresponding changes in the breadth of the dielectric dispersion (if the polymer is amorphous) and in the $T_{\mathrm{g}}$-scaled temperature dependence of the relaxation time. Although these changes have been verified, within a class of polymers the magnitude of the changes may not be large (see Figures 1-5). To see a much larger variation in cooperativity plots, in Figure 6 we combine the data from these disparate classes. The $T_{\mathrm{g}^{-}}$ scaled temperature dependencies now vary greatly, increasing from the weak and almost Arrhenius behavior of the simplest polyoxide, POM, and the simplest hydrocarbon, PE, to the moderate dependence exhibited by PAA, PPO, PVME, PVAc, and other vinyl polymers, to the 


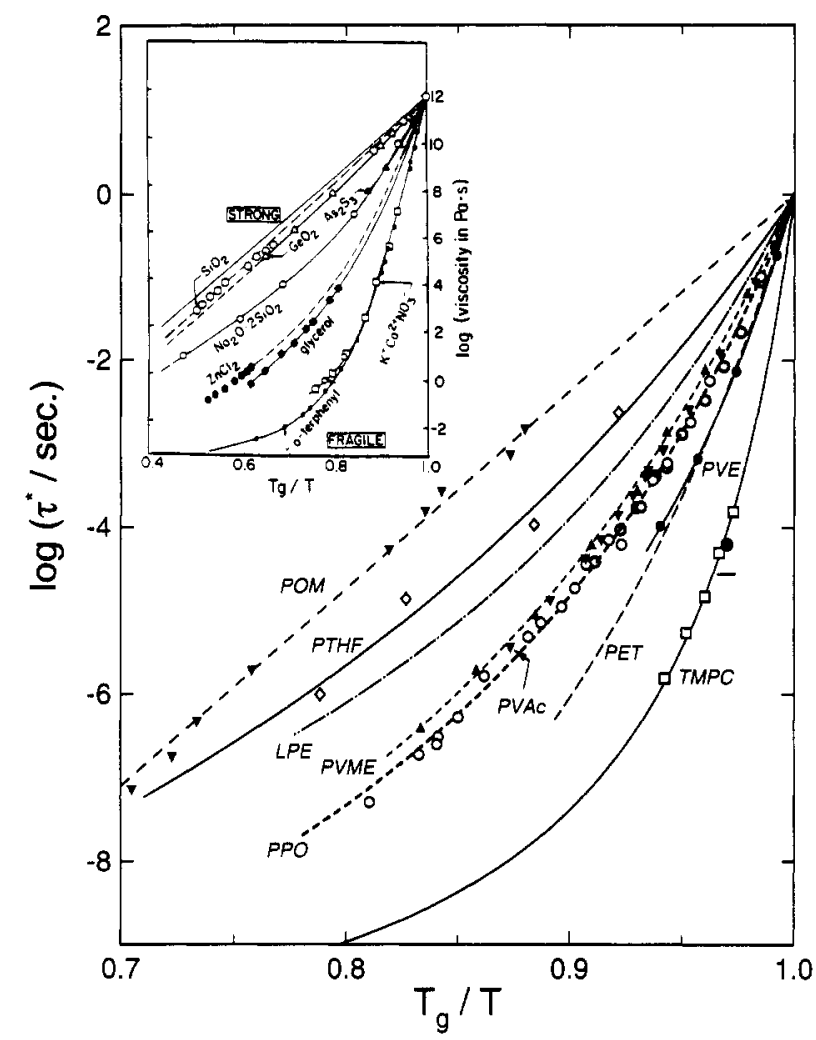

Figure 6. Cooperativity plots for a variety of polymers, representing the extremes from strong intermolecular coupling in tetramethyl polycarbonate to the almost Arrhenius behavior of poly(oxymethylene). The inset shows the original $T_{\mathrm{g}}$-scaled plots of shear viscosity of Angell ${ }^{43}$ for small molecular and network glass-forming liquids.

stronger dependence of PVE and PET, and finally to the strongest dependence observed in the polycarbonates. For the four polymers PAA, PPO, PVME, and PVAc having nearly the same $T_{\mathrm{g}}$-scaled temperature dependence, we show in Figure 7 that their dielectric dispersions are also quite similar. Figure 7 is a plot of the dielectric loss data of these four amorphous polymers after the peaks have been normalized to the same height and shifted horizontally to occur at the same frequency.

In an inset of Figure 6 we show Angell's original $T_{g-}$ scaled plot of shear viscosity of small molecule and network glass-forming liquids. ${ }^{43}$ By comparing this inset with the main result for the polymers, we can conclude that the patterns seen for small molecule and network glass-forming liquids are reproduced in polymers, both amorphous or semicrystalline. It is gratifying to find that the limiting Arrhenius behavior seen in $\mathrm{SiO}_{2}$ and $\mathrm{GeO}_{2}$ for nonpolymeric glass-forming liquids is recaptured for the polymer systems by the oxide polymers. Also there are polymers like BPA-PC and TMPC that have considerably stronger temperature dependence than any of the smallmolecule liquids.

It is difficult to use comparisons of chemical structure to predict changes in intermolecular coupling for all the glass-forming liquids shown in the inset of Figure 6 . These materials range from inorganic networks, to molten salts, and to small molecular van der Waal liquids. Meaningful comparisons can only be made if we restrict consideration to within some subclass of materials, such as the small molecule glass-forming liquids. In Figure 8 we illustrate the structures of some of these, along with the $T_{\mathrm{g}}$-scaled temperature dependence of their dielectric relaxation time (obtained from published data ${ }^{10,61}$ ). Here $T_{\mathrm{g}}$ is again defined as the temperature at which the dielectric relaxation time equals $1 \mathrm{~s}$. There are remarkable similarities between the features shown in Figures 6 and 8. 3-Bro-

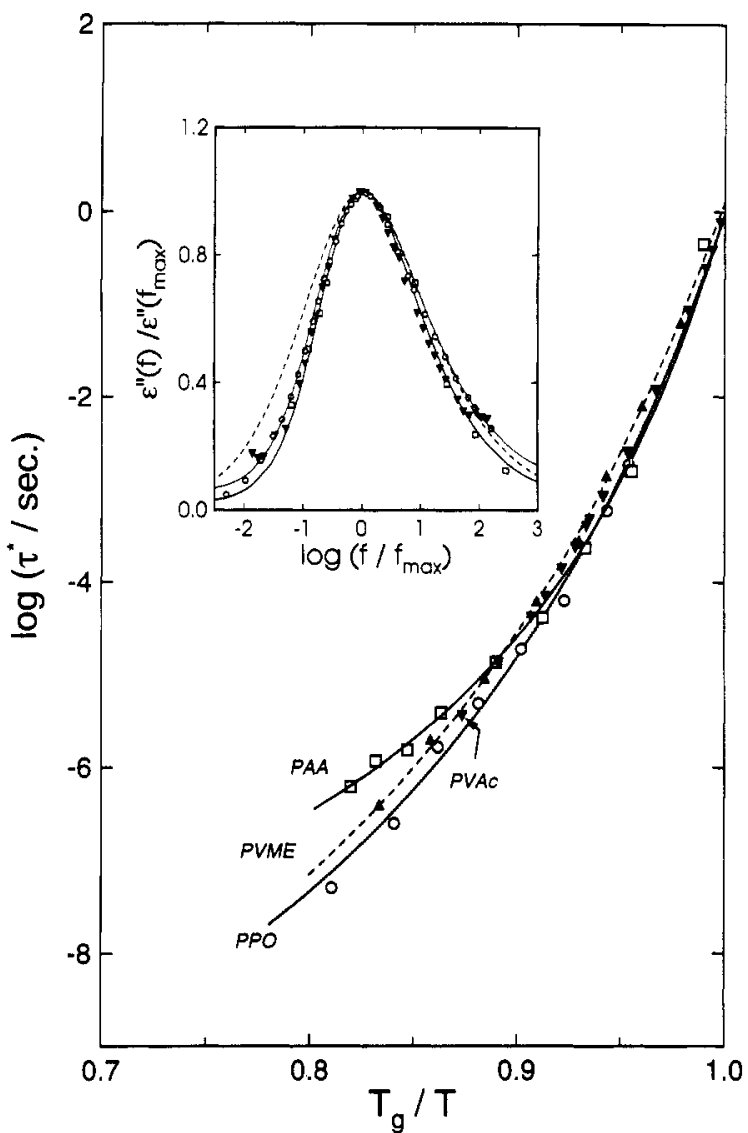

Figure 7. Dielectric loss data of four polymers having nearly the same $T_{\mathrm{g}}$-scaled temperature dependencies. The peaks have been normalized to the same height and shifted horizontally to occur at the same frequency. A similar degree of intermolecular cooperativity for their segmental relaxations is reflected in the similar time and temperature dependencies.

mopentane is closely related to LPE, except the introduction of bromine into the hydrocarbon, which may confer some polar character to the intermolecular forces. Propylene glycol is generically related to PPO. Salol and orthoterphenyl are small molecules that contain the phenyl ring or rings and can be considered as analogues of polymers that have phenyl rings in their monomer units (e.g., polystyrene, PET, and BPA-PC). The rank ordering of the cooperativity plots (Figure 8 ) for these small-molecule liquids follows the same trend as their polymeric analogues in Figure 6. This trend among the small-molecule liquids can be correlated with the capacity for intermolecular coupling deduced from their molecular structures. In the inset of Figure 8 the KWW fits to the dielectric loss data of propylene glycol, 3-bromopentane, and salol are compared. The breadth of the loss spectrum, and hence the coupling parameter itself, increases when going from propylene glycol to 3 -bromopentane and salol, a trend that can be rationalized from the change in chemical structure in the same manner as we have done above for polymers.

\section{Summary}

The analysis presented herein provides a physical basis for the coupling parameter describing segmental relaxation. The magnitude of this coupling parameter can be related in a plausible fashion to the polymer's chemical structure; specifically, polymers with smoother, less polar, more compact, symmetric or flexible-chain backbones and/ or having less sterically-hindering pendant groups experience weaker constraints on their segmental relaxation from interactions with neighboring, nonbonded segments. Note that the correlation developed herein interprets the behavior in terms of steric effects and backbone structure; 


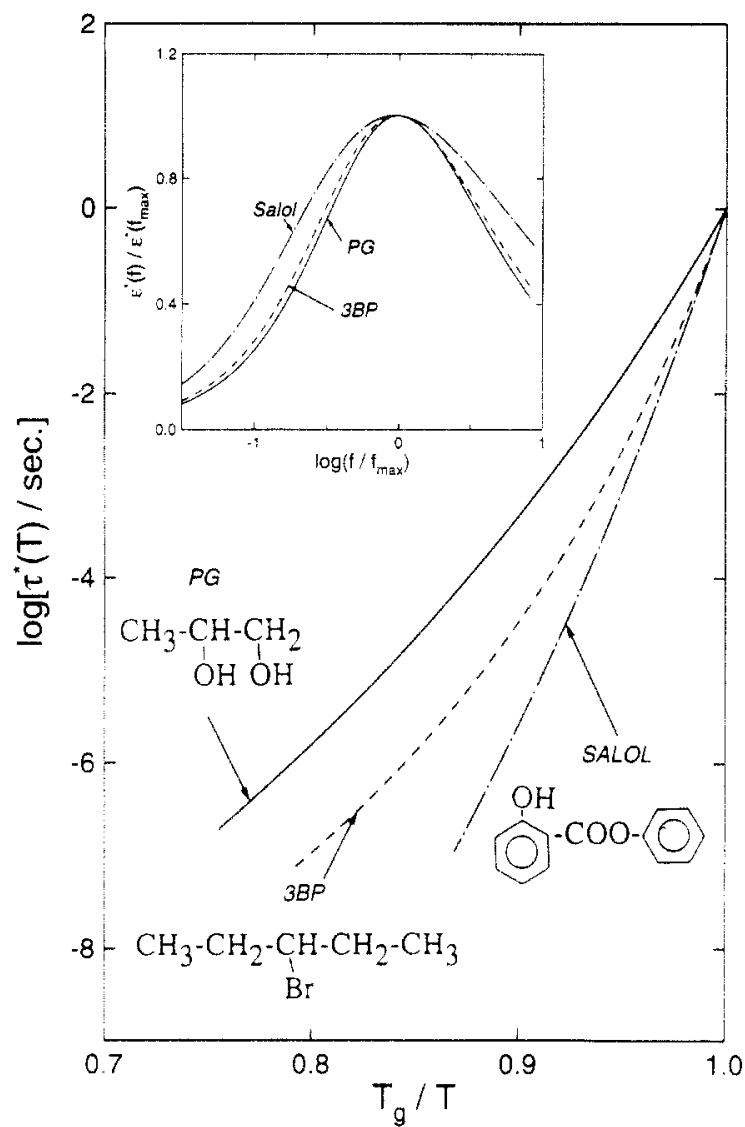

Figure 8. $T_{\boldsymbol{g}}$-scaled temperature dependence of the dielectric relaxation time for three small-molecule glass formers, propylene glycol, 3-bromopentane, and salol $\left(T_{\mathrm{g}}=173,181\right.$, and $222 \mathrm{~K}$, respectively). The breadth of their respective dielectric dispersions (inset) correlates with the steepness of the cooperativity curves. Both properties can be rationalized in terms of the effect of chemical structure on the strength of the intermolecular coupling.

no recourse to free volume considerations is necessary. The extension of the previously adduced correlation of time and temperature dependencies of segmental relaxation to a correlation with chemical structure will be useful in the future for sorting out the dependence on chemical structure of the viscoelastic properties of polymers in the glass-rubber transition zone. The results presented herein represent a continuing effort to understand the manner in which chemical structure underlies viscoelastic behavior.

Acknowledgment. This work was supported by the Office of Naval Research. K.L.N. is supported in part by ONR Contract No. N0001493WX24011. Time domain dielectric spectra on PVE were obtained by Mr. J. Driscoll of Imass, Inc., to whom the authors express their appreciation.

\section{References and Notes}

(1) Boyer, R. F. Rubber Chem. Technol. 1963, 36, 1303.

(2) Eisenberg, A.; Shen, M. Rubber Chem. Technol. 1970, 43, 156.

(3) Matsuoka, S.; Quan, X. Macromolecules 1991, 24, 2770.

(4) Matsuoka, S.Relaxation Phenomena in Polymers; Hanser: New York, 1992; Chapters 2 and 3.

(5) Angell, C. A. In Relaxations in Complex Systems; Ngai, K. L., Wright, G. B., Eds.; Government Printing Office: Washington, DC, $1985 ; 03$

(6) Ngai, K. L. J. Non-Cryst. Solids 1987, 95-96, 969.

(7) Ngai, K. L.; Rendell, R. W.; Plazek, D. J. J. Chem. Phys. 1991, 94, 3018.

(8) Angell, C. A. J. Non-Cryst. Solids 1991, 131-133, 13.
(9) Torell, L. M.; Grimsditch, M. Proc. Phys. 1989, 37, 196.

(10) Dixon, P.; Wu, L.; Nagel, S.; Williams, B. D.; Carini, J. P. Phys. Rev. Lett. 1990, 65, 1108.

(11) Plazek, D. J.; Ngai, K. L. Macromolecules 1991, 24, 1222

(12) Roland, C. M.; Ngai, K. L. Macromolecules 1991, 24, 5315; 1992, $25,1844$.

(13) Roland, C. M.; Ngai, K. L. Macromolecules 1992, $25,363$.

(14) Ngai, K. L.; Roland, C. M.; O'Reilly, J. M.; Sedita, J. S. Macromolecules 1992, 25, 3906.

(15) Roland, C. M.; Ngai, K. L. Macromolecules 1992, 25, 5765.

(16) Roland, C. M. Macromolecules 1992, 25, 7031.

(17) Tobolsky, A. V. Adv. Polym. Sci. 1969, 6, 103.

(18) Choe, S.; Aklonis, J. J. Polym. Sci. Eng. 1987, 27, 1284.

(19) Chang, E. P.; Lin, K. S.; Kaelble, D. H. J. Polym. Sci., Polym. Phys. Ed. 1985, 23, 825 .

(20) Tribone, J. J.; O'Reilly, J. M.; Greener, J. Macromolecules 1986 , $19,1732$.

(21) Kelchner, R. E.; Aklonis, J. J. J. Polym. Sci., Part A 1970, 8 , 799.

(22) Hall, C. K.; Helfand, E. J. Chem. Phys. 1982, 77, 3275.

(23) Helfand, E. Science 1984, 226, 647.

(24) Dejean de la Batie, R.; Laupretre, F.; Monnerie, L. Macromolecules 1988, 21, 2045.

(25) Bahar, I.; Erman, B.; Monnerie, L. Macromolecules 1991, 24, 3618.

(26) Ngai, K. L.; Rendell, R. W.; Rajagopal, A. K.; Teitler, S. Ann. N.Y. Acad. Sci. 1986, 484, 150.

(27) Ngai, K. L.; Rajagopal, A. K.; Teitler, S. J. Chem. Phys. 1988, 88,6088 .

(28) Ngai, K. L.; Rendell, R. W. J. Non-Cryst. Solids 1991, 131-133, 942.

(29) Ngai, K. L.; Peng, S. L.; Tsang, K. Y. Physica A 1992, 191, 523.

(30) Bahar, I.; Erman, B.; Kremer, F.; Fischer, E. W. Macromolecules $1992,25,816$.

(31) Takeuchi, H.; Roe, R.-J. J. Chem. Phys. 1991, 94, 7446.

(32) Roe, R.-J.; Rigby, D.; Furuya, H.; Takeuchi, H. Comput.Polym. Sci. 1992, 232.

(33) Kohlrausch, R. Pogg. Ann. Phys. 1847, 12, 393.

(34) Williams, G.; Watts, D. C. Trans. Faraday Soc. 1970, 66, 80.

(35) Comenero, J.; Arbe, A.; Alegria, A. Phys. Rev. Lett, in press.

(36) Roe, R.-J., submitted for publication in J. Chem. Phys.

(37) McCrum, N. G.; Read, B. E.; Williams, G. Anelastic and Dielectric Effects in Polymer Solids; Wiley: London, 1967.

(38) Wetton, R. E.; Allen, G. Polymer 1966, 7, 331.

(39) Sawada, K.; Ishida, Y. Rep. Prog. Polym. Phys. Jpn. 1974, 17 437.

(40) Boyd, R. H. Polymer 1985, 26, 323

(41) Ngai, K. L.; Roland, C. M. Macromolecules, in press.

(42) Laughlin, W. T.; Uhlmann, D. R. J. Chem. Phys. 1972, 76, 2317.

(43) Angell, C. A. In Relaxations in Complex Systems; Ngai, K. L., Wright, G. B., Eds.; Government Printing Office: Washington, DC, $1985 ;$ p 3 .

(44) Angell, C. A. J. Non-Cryst. Solids 1991, 131-133, 13

(45) Allen, G.; Higgins, J. S.; Maconnachie, A.; Ghosh, R. J. Chem. Soc., Faraday Trans. 2 1982, 78, 2117.

(46) Colmenero, J.; Alegria, A.; Arbe, A.; Frick, B. Phys. Rev. Lett. $1992,69,478$.

(47) Ngai, K. L.; Colmenero, J.; Alegria, A.; Arbe, A. Macromolecules $1992,25,6727$

(48) Williams, G. Trans. Faraday Soc. 1965, 61, 1564

(49) Ngai, K. L.; Schlosser, E.; Schonhals, A. Macromolecules 1992 25,4915 .

(50) Connor, T. M.; Read, B. E.; Williams, G. J. Appl. Chem. 1964 14,74 .

(51) Ngai, K. L.; Roland, C. M., to be published.

(52) Ishida, Y.; Matsuo, M.; Yamafuji, K. Kolloid Z. Z. Polym. 1962 180,108

(53) Nozaki, R.; Mashimo, S. J. Chem. Phys. 1986, 84, 3575.

(54) Rendell, R. W.; Ngai, K. L.; Mashimo, S. J. Chem. Phys. 1987, $87,2359$.

(55) Floudas, G.; Higgins, J. S.; Kremer, F.; Fischer, E. W. Macromolecules $1992,25,4955$.

(56) Colmenero, J.; Alegria, A.; Roland, C. M.; Ngai, K. L., to be published.

(57) Zetsch, A.; Kremer, F.; Jung, W.; Schulze, H. Polymer 1990, 31 , 1883.

(58) Ishida, Y.; Matsuoka, S. Polym. Prepr. (Am. Chem. Soc., Div. Polym. Chem.) 1965, 6 (No. 2), 795.

(59) Saito, K. Res. Electrotech. Lab. (Tokyo) 1964, No. 648.

(60) Roland, C. M.; Ngai, K. L.; O'Reilly, J. M.; Sedita, J. S. Macromolecules 1992, 25, 3906

(61) Berberian, J. G.; Cole, R. H. J. Chem. Phys, 1986, 84, 6921. 